\title{
Adaptive Programming of Unconventional Nano-Architectures
}

\author{
John W. Lawson* and David H. Wolpert \\ Mail Stop 269-2 \\ Center for Nanotechnology \\ NASA Ames Research Center \\ Moffett Field, CA 94035
}

\begin{abstract}
Novel assembly processes for nanocircuits could present compelling alternatives to the detailed design and placement currently used for computers. The resulting architectures however may not be programmable by standard means. In this paper, nanocomputers with unconventional architectures are programmed using adaptive methods. The internals of the device are treated as a "black box" and programming is achieved by manipulating "control voltages". Learning algorithms are used to set the controls. As examples, logic gates and simple arithmetic circuits are implemented. Additionally, similar methods allow for reconfiguration of the devices, and makes them resistant to certain kinds of faults.
\end{abstract}

Keywords: Nanoelectronics, Computer Architectures, Circuit Optimization, Fault Tolerance, Machine Learning, Learning, Adaptive Methods, Optimization, Programmable Circuits, Control Theory

*Electronic address: John.W.Lawson@nasa.gov

${ }^{\dagger}$ Electronic address: David.H.Wolpert@nasa.gov 


\section{INTRODUCTION}

The long-standing trend of miniaturizing electronic components is expected to encounter serious obstacles in the not too distance future. Despite the impressive successes in designing electronic components down to the atomic and molecular scales, serious difficulties are anticipated in assembling them into realistic functional architectures using current technologies. One such issue is the expected, excessive cost of both designing and reliably manufacture chips on such small scales using conventional methods. It is possible, in fact, that a major limiting factor in future efforts at miniaturization won't be related to science or technology issues, but rather to the high cost of the associated manufacturing processes.

Development of novel approaches for assembling nanochips may be a critical ingredient for this new technology. One possibility, for example, could be to rely on chemical synthesis methods (self-assembly, etc...) to assemble individual components (which may be individual molecules) into larger functional unit $\frac{1}{}$. Chemical synthesis methods are capable of organizing large numbers of atoms and molecules into large, regular, ordered structures. This might be the basis for a relatively cheap and efficient assembly process. What is not clear, however, is if any of the resulting structures would correspond to something resembling a computer chip. Would we know how to program it, for example?

Another potential source of difficulty is the degree of randomness inherent in nanosystems. Traditional computer chips are designed and manufactured very meticulously, and therefore, are very sensitive to the presence of defects or disorder. With nanocomputers, the individual components themselves are expected to be extremely sensitive to disorder, and this may have a serious impact on their reliability. Randomness in single electron transistors,

for example, may be unavoidable ${ }^{2}$. The effects of integrating such components into a larger circuit are only beginning to be considered.

If we are to take advantage of novel assembly methods and potentially exotic architectures, we will need to learn how to program the resulting devices. Some assembly methods may give very ordered, predictable structures. Other techniques may be more extreme and produce a range of essentially random configurations. In this paper, we consider general purpose methods to program such devices that are independent of the internal structure, and therefore also of the assembly process. 
Minimally, the devices we consider have input and output leads as well as a set of additional leads we designate as "controls". We assume that the internal components are sufficiently well-connected to allow signals to propagate across the device. Beyond that we consider the internal structure as unknown. By adjusting voltages on the controls leads, we manipulate the outputs that appear for a given set of inputs. In particular, we use adaptive methods to find the set of control voltages that implement a desired function. In this way, we program the device.

To develop and test these methods, we introduce a simple, abstract model that we call a randomly assembled computer (RAC). In this model, we place $N$ two-states devices ("diodes") randomly on a chip and connect them together with random strengths. We designate a subset of the diodes as inputs, outputs, and controls and attempt to program the RAC by manipulating the controls. We phrase the programming of such a device as an optimization problem. We define an error function that measures the difference between the function currently implemented by the RAC and some desired input-output function $f$. Since this error function $E_{f}(\vec{c})$ depends on the values of the controls, we seek a control vector $\vec{c}$ that minimizes the error. The task is to find a $\vec{c}$ such that $E_{f}(\vec{c})=0$. In general, there may be many solutions to this equation, or possibly, none at all, for a particular $f$ and a particular RAC.

It is important to emphasize that our main interest is in developing programming algorithms. We are not advocating a particular hardware architecture or assembly process. A $\mathrm{RAC}$ is therefore only a test bed for our optimization methods. In fact, we expect that in the real world, there will be a large amount of information available about the internal structure of the device from the assembly process itself. For example, in a chemical assembly process, the device might be imaged. This information could be exploited to facilitate programming/optimization. Additionally, different degrees of programming can be imagined. For example, for very predictable, repeatable assembly processes, adaptive programming may only be needed once for an entire class of devices. On the other hand, for devices with more random internal structures, individual programming may be necessary. However, even in this more extreme case, devices could be programmed in parallel, or possibly be connected together in large number where they could program each other. In this work, however, we do not take explicit advantage of such information. Thus the problem we set for ourselves 
may be more challenging that what will be faced in the real world. However, it will provide a good testing ground for our algorithms.

Related work has included ideas using neural nets ${ }^{3,4}$, as well as the Nanocell architecture $\mathrm{F}^{\frac{5}{}}$. The Nanocell approach has some ideas in common with ours, however, in that approach a particular architecture based on molecular components is presented, while we aim to present methods that are independent of a particular architecture. Furthermore, many of those results depended on detailed knowledge and control of the full internal states of the Nanocell. This permitted a "proof of concept" to demonstrate that internal states do indeed exist that correspond to certain simple functions. The broader issue of how to find those states was only hinted at, however. Here, this issue of "black box programming" is our main concern.

In this paper, we consider different optimization methods to program randomly assembled computers. In particular, we will employ simulated annealing as well as adaptive, multi-agent methods. We will see that while simulated annealing is adequate for small systems, larger devices require more sophisticated approaches. We have found in previous work that adaptive multi-agent methods perform very well for large scale optimization problems ${ }^{6}$. Other optimization methods could be considered as well including genetic algorithms, cellular automata, etc. These approaches may have advantages in certain situations, but we found our methods to be more than adequate for our purposes. We will be primarily concerned with two general questions. First, in what generality can RACs be programmed i.e. what is the range of functionality that can be implemented on a given RAC? And secondly, what methods might be appropriate to perform the programming?

\section{RANDOMLY ASSEMBLED COMPUTERS}

We consider a RAC as having $P$ input variables $\vec{I}=\left(I_{1}, \ldots, I_{P}\right), M$ output variables $\vec{O}=\left(O_{1}, \ldots, O_{M}\right)$, and $K$ control variables $\vec{c}=\left(c_{1}, \ldots, c_{K}\right)$. A schematic of a RAC can be seen in Figure 11. Here input and output variables are binary-valued, while the controls can take continuous values. Our goal is to find a $\vec{c}$ that implements a desire mapping

$$
f: \vec{I} \rightarrow \vec{O}
$$


We specify a "target" function $f$ as the ordered set of outputs associated with all possible input vectors, and where $\vec{T}=\left(T_{1}, \ldots, T_{M}\right)$ are the target outputs.

A RAC is properly "programmed" when $\vec{c}$ causes the RAC outputs $\vec{O}$ to equal the targets $\vec{T}$ over a "training set" of $L$ input/output pairs $\left\{\vec{I}^{l}, \vec{T}^{l}\right\}$ representing $f$. We quantify how well we have programmed a RAC thru an error function defined as

$$
E \equiv E_{f}(\vec{c})=\frac{1}{M L} \sum_{l=1}^{L}\left\|\vec{O}^{l}-\vec{T}^{l}\right\|
$$

where $\vec{O}^{l}$ is shorthand for $\vec{O}_{\vec{c}}\left(\vec{I}^{l}\right)$ and $\left\|\vec{O}^{l}-\vec{T}^{l}\right\|$ is the number of components in which $\vec{O}_{\vec{c}}^{l}\left(\vec{I}^{l}\right)$ and $\vec{T}^{l}$ disagree. Thus, $E$ is the fraction over all output variables and training set elements of mistakes made by the RAC. Programming a RAC corresponds to minimizing $E$. In general, the results we will present will be averages over large ensembles of RACs.

To study different programming methods, the following model system was used $\underline{\underline{Z}}$. The internal structure of a RAC was represented by randomly placing $N$ "diodes" $d_{i}(t), i=$ $1, \ldots, N$ (i.e., two-state devices such that $d_{i} \in[0,1]$ ) on a chip, and connecting them together with random strengths. An arbitrary subset of diodes was designated as inputs, and another subset (perhaps overlapping) as outputs. Diodes can change state based on their individual inputs at discrete time steps $t$ governed by a global clock. Thus, a RAC has the structure of an iterated function $\vec{d}(t+1)=F[\vec{d}(t)]$. A computation begins at $t=0$ by setting each input diode $d_{p^{\prime}}(0)=I_{p}$ where $I_{p}$ is the input to the RAC and $p^{\prime}$ is the diode associated with input bit $p$. All other diodes are initialized to zero. At subsequent time steps, the diodes update their states according to the rule

$$
d_{i}(t+1)=g\left(\sum_{j}^{N} \omega_{i j} d_{j}(t)+\sum_{j}^{K} \rho_{i j} c_{j}(t)\right)
$$

where the $\omega_{i j}, \rho_{i j}$ are pre-fixed random numbers, and $g(x)=\frac{1}{2}(1+\tanh (\beta x))$ is a smoothed step function with "gain" $\beta$ that models the switching behavior of the diodes. Here, the $\omega_{i j}$ represent the strength of the connection between two diodes, $i$ and $j$, analogous to a resistance. Note also that for low values of the gain, the diodes can take on values between zero and one, given by the function $g(x)$. After a fixed number of time steps $t=T$, the outputs can be read to give the result of the computation $O_{m}=d_{m^{\prime}}(T)$, where $m^{\prime}$ is the diode associated with output bit $m$. For simplicity, we consider only circuits composed of diodes and do not include other possible components such as memory elements, etc. 
It should be noted that our model is used to test our programming approach. However, none of the adaptive programming methods that we present here depend on the particular details of the model, and are expected to be general enough to work for any "black box" nano-

circuit. Moreover, our model captures several important features of real circuits consisting of an iterated network of diodes whoses states can change depending on their local environment. It is also important to note that while the $d_{i}(t)$ evolve according to a dynamical rule, Equation 3, the $c_{i}(t)$ are external, and potentially time-dependent parameters. Our task, in fact, will be to set these parameters.

\section{MULTI-AGENT METHODS}

We want to find the best $\vec{c}$ that minimizes $E_{f}(\vec{c})$ for a given training set. The best case scenario is to find a $\vec{c}$ that gives $E_{f}(\vec{c})=0$, i.e., find a $\vec{c}$ so that the RAC gives the correct outputs for the given inputs over all the examples. We will see that this is often achievable. Several issues make this a potentially difficult optimization problem. First, $E$ is a very nonlinear function with a potentially large number of local minima. Secondly, the exact functional form of $E_{f}(\vec{c})$ is not known due to our desire to treat the RAC as a black box. The only information that we have available is the set of input/outputs pair values $\left(\vec{I}^{l}, \vec{O}^{l}\right)$ for a given control $\vec{c}$. Lastly, there may be a large number of controls that need to be set. Because of these difficulties, we will employ a set of methods recently developed in the context of multi-agent systems. These multi-agent methods have some relation to simulated annealing (SA), but have significant differences, and have shown dramatically faster convergence in a number of problems.

There are three main differences between the multi-agent methods we will consider and

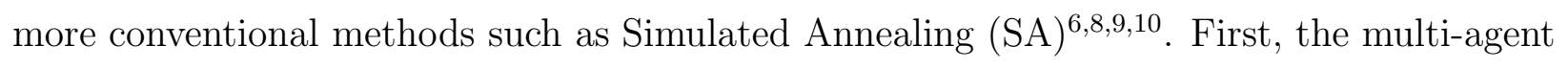
approach is distributed. Thus, instead of considering a $N$-dimensional optimization problem, we deal with $N$ 1-dimensional ones. Each independent variable $c_{i}$ is regarded as an agent, and each agent $i$ separately sets its variable $c_{i}$ in order to try to optimize an associated objective function $e_{i}(f, \vec{c})$. Thus, we have $N$ independent optimization processes, each of which we are calling an "agent". In general, distributed approaches such as these are expected to scale better for large problems. 
Secondly, each agent solves its particular optimization problem using Reinforcement Learning $(\mathrm{RL})^{11}$. The version of $\mathrm{RL}$ we use is called Boltzmann learning and is related to SA. The principal difference is that instead of taking "random" trial steps, RL relies on previous data to make "smart" trial steps. In this way, RL algorithms converge very rapidly. Boltzmann learning is also easy to implement. More sophisticated algorithms may be necessary, however, for more difficult problems.

In our RL algorithm, at each simulation time step $\tau$, each agent $i$ randomly generates a number of candidate values for $c_{i}(\tau+1)$ from some $\Delta$ neighborhood of the current $c_{i}(\tau)$. Next, the agent estimates, based on data from previous time steps, a value of $e_{i}$ for each candidate value. This is done by performing a weighted average over all previous pairs $\left(c_{i}\left(\tau^{\prime}\right), e_{i}\left(\tau^{\prime}\right)\right)$, for $\tau^{\prime}<\tau$. The weighting damps the contribution from "old" data (i.e., from $c_{i}\left(\tau^{\prime}\right)$ and $e_{i}\left(\tau^{\prime}\right)$ where $\left.\tau^{\prime}<<\tau\right)$. Finally, a Boltzmann probability distribution over these estimates is sampled to select the resulting trial move. A nonzero simulation temperature prevents us from getting stuck in local minima.

After the agents have all made their moves, it may turn out that the global error $E$ has actually increased. This may happen if, for example, the agents' estimates are inaccurate. To address this, we also include a Metropolis-style global accept/reject step. If after the agents have made their moves $E$ has decreased, then we accept the new $\vec{c}$. Otherwise we reject it with conditional probability proportional to $e^{-\beta\left(E-E^{\prime}\right) \underline{\underline{6}}}$.

The third difference with conventional methods is that the individual agents may be assigned different objective functions. Since our global objective is to minimize $E$, we might expect that each agent should use $E$ as its individual objective function, $e_{i}=E$. Such a system of agents who all have the same objective function is called a Team Game (TG), in analogy with the scenario in game theory where all the players/agents have the same payoff function. We expect — and indeed observe — that for small systems Team Games outperform SA. This is because by using learning algorithms, the agents can make "smart" trial moves inferred from their past history, as opposed to the random ones with conventional SA.

However, as the system size grows, it becomes more difficult for each individual agent to discern its impact on $E$, and thus it becomes more difficult for each agent to choose an optimal value if $e_{i}=E$. To address this we define, heuristically, a signal-to-noise measure 
for each agent $i$ that we call "learnability":

$$
\lambda_{i}^{e_{i}}=\frac{\left|\partial_{i} e_{i}\right|}{\left|\vec{\nabla}_{\hat{\imath}} e_{i}\right|}
$$

where $\partial_{i} e_{i}=\partial e_{i} / \partial c_{i}$, and $\vec{\nabla}_{\hat{i}} e_{i}$ denotes the gradient of $e_{i}$, but with the component in the $i^{\text {th }}$ direction removed. The learnability of agent $i$ measures the sensitivity of $e_{i}$ to changes in $c_{i}$ relative to changes in the system as a whole. It is expected that for large $\lambda_{i}$, agent $i$ can more easily discern its impact on $e_{i}$, and therefore, it can make better trial moves. On the other hand, we expect that beyond a certain system size, the denominator of $\lambda_{i}^{e_{i}}$ will grow to such a point that the agents will be essentially making random moves. At that point, Team Games and SA will basically give the same result.

Even though our goal is for the system as a whole to minimize the error $E$, there is nothing to prevent us from giving different $e_{i}$ to the different agents. To exploit this, each agent is assigned a "difference utility" $e_{i}$ of the form

$$
e_{i}(\vec{c})=E(\vec{c})-\left.E(\vec{c})\right|_{c_{i}=0}
$$

Since $\partial_{i} e_{i}=\partial_{i} E$, critical points of $e_{i}$ will be critical points of $E$. Thus, if agent $c_{i}$ optimizes $e_{i}$, it will optimize $E$ as well. The functions $e_{i}$ that are aligned with $E$ in this manner are called factored.

In addition to being factored, $e_{i}$ also typically has better learnability than $E$. This is because the numerators of $\lambda_{i}^{E}$ and $\lambda_{i}^{e}$ are identical, but the denominators are very different. In fact, we expect in general that $\left|\partial_{j} e_{i}\right|<<\left|\partial_{j} E\right|$. This should lead to a substantial reduction in the background noise, and hence an increase in $\lambda_{i}$. This behavior is born out by many simulations. Such $e_{i}$ that are both "factored" and have high "learnability" are expected to perform well for large systems.

Notice that equation (5) is only one choice for a difference utility. In previous work, this utility was called the Wonderful Life Utility (WLU). The value for $c_{i}$ in the subtracted term could have been set to a different, non-zero, value, and the advantages would still hold. A similar possibility is to subtract an expectation value taken over all $c_{i}$ weighted by an appropriate probability density.

$$
e_{i}(\vec{c})=E(\vec{c})-<E(\vec{c})>_{c_{i}}
$$


Previously, this utility has been called the Aristocrat Utility (AU). Both WLU, AU, and other utilities have been studied extensively ${ }^{10}$. In this paper, we will concentrate on WLU for simplicity. A fully formal derivation of WLU and AU based on bias and variance may be found in ${ }^{12.13}$. This work has many other advantages beyond that presented here, e.g., explicit connection to bounded rational game theory and statistical physics. However it is more involved than is needed for current purposes.

\section{RESULTS}

We performed simulations on a variety of target functions $f$, and on RACs of different sizes $(\mathrm{N}, \mathrm{K})$, where $\mathrm{N}$ is the number of diodes and $\mathrm{K}$ is the number of controls. Once a RAC was assembled and a function selected to be programmed, an error function could be defined. Depending on the size of the RAC, we use different optimization algorithms to program them. For smaller RACs $(N, K<20)$, SA often proved adequate, but for larger RACs $(N, K>50)$, multi-agent methods were essential.

To begin, we first considered 2-input, 1-output logic gates: INV, AND, OR, and XOR programmed using simulated annealing for the optimization. For these simple cases, SA performs adequately, and in general, runs faster (in minutes as opposed to hours on a workstation) than the multi-agent approaches. We demonstrate that not only can these functions indeed be programmed, but we attempt to answer an important general question which is what is the range of functionality that a given RAC can implement. Indeed, we expect that some functions cannot be implemented at all on a particular RAC. We might hope, however, that a variety of functions can be programmed on a RAC of sufficient size. That minimal size will depend on the particular function being considered. Thus, even for logic gates, we might ask: how big of a RAC is needed to have a high programming success rate?

The results for certain logic gates are shown in Figure 2. Each data point represents the result of programming attempts on 10,000 different RACs of the given size $(N, K)$. The $\mathrm{y}$-axis gives the fraction that were successfully programmed i.e. all the outputs matched the targets with no mistakes $(E=0)$. Different curves correspond to different proportion of controls to diodes. We see that the Inverter (INV) with $K=N$ has a greater than $90 \%$ 
success rate for $N \geq 12$ whereas the $\mathrm{XOR}$ required $N \geq 18$. This is expected due to the greater complexity of the XOR. Furthermore, we see that reducing the number of controls by half has only a relatively small effect on the performance, with the success rate still better than $90 \%$, but for $N \geq 30$. Lastly, we see, even with a fixed number of controls $(\mathrm{K}=2)$, a large fraction ( $50 \%$ for the INV and $30 \%$ for the XOR) can still be programmed.

Next, we considered larger circuits and larger RACs. We implemented larger functional units, namely arithmetic circuits. In particular, we examined 2-bit adders with carry and multipliers. These are significantly more complicated functions than the 2-input logic gates. Our adders and multipliers performed operations on two 2-bit numbers, giving a total of 4 input bits, and 3 output bits. With 4 inputs, there are 16 possible input combinations, and in total, $2^{3 \times 2^{4}}=2^{48}$ possible binary functions from 4 inputs to 3 outputs. An adder or a multiplier is only one of these possible functions. Our optimization algorithm was required to find these particular functions out of the large number of possibilities. For these functions, with 16 possible inputs and 3 bit output, there are 48 total output bits that must be correctly set in order for the RAC to have been programmed perfectly. The truth table in Table 1 gives the required input/output combinations that define these functions.

For functions of the complexity of adders and multipliers, we used RACs of size $(\mathrm{N}=100, \mathrm{~K}=40)$ with $(\mathrm{T}=5)$ iteration cycles. For a dynamically controlled RAC (i.e. the control voltages change with each clock cycle $\vec{c}(t))$, this results in 200 control parameters. For such a large number of parameters, multi-agent methods were essential.

The upper plots in the Figures 3 , 4for the adder and the multiplier show the convergence of different methods as a function of simulation time. In each case, the error function $E_{f}$ is averaged over an ensemble of 1000 RACs. We see for both the adder and the multiplier, that for a RAC of this size, the Team Game (TG) behavior is only marginally better than SA, while the multi-agent WLU does considerably better due to its superior scaling properties. In the lower plots, we deconstructed the convergence graphs and show how well the individual RACs were programmed. Each data point gives the fraction of RACs that made a given number of mistakes after programming was completed. The ideal situation would be a fraction of 1 (all the RACs) making 0 mistakes; this would correspond to all the RACs being perfectly programmed. We see that WLU programmed RACs made much fewer mistakes than SA programmed ones. In fact, for the adder, almost $50 \%$ of the RACs programmed 
with WLU made only 0 or 1 mistake out of 48. In no case, were there more 10 mistakes with WLU. On the other hand, the best performing SA RAC still made 9 mistakes. For the multiplier, more that $20 \%$ of the WLU RACs make no mistakes while the best for SA is at 4 mistakes.

For these larger circuits, we did additional runs where we show how including additional information or control over the circuit can affect the performance. In programming unconventional circuits, some information may be available about the internal structure. For example, with a random assembly process, large batches of chips might be produced very inexpensively due to the negligible design and placement costs. It would be very easy therefore to quickly generate and test a subset of these devices to see which functions they implement without any programming all, and then pick the ones whose initial error was the lowest for subsequent programming. Clearly, it would more efficient to program a RAC that is closer originally to the desired function. To model this possibility, we generated $100 \mathrm{RACs}$, and programmed the one with the lowest initial error. Additionally, it may also be possible that during the programming process, we may have control over some physical parameters of the device such as an external field. To model this scenario, we adjust the gain parameter $\beta$ of our model during the programming process. In particular, we found an improvement in performance by annealing the gain. Sets of runs with these additional features are labeled "Opt" in Figures 3, 4, In all cases, the "Opt" runs improved performance.

The random assembly of the RAC and the adaptive nature of the multi-agent methods makes them well-suited for handling faults, defects, noisy components, and also allows them to be redesigned/reused. To illustrate this flexibility, we performed the following simulations. First, to illustrate RAC reuse/reconfigurability, we initially programmed an ensemble of RACs with $(N=20, K=10)$ to implement AND functions. From Figure reffig:inv, we expect a $90 \%$ success rate across the ensemble. Then, at simulation time step $\tau=3000$, we abruptly changed the same RAC to implement a different function, namely an XOR. As can be seen in the top plot of Figure [5 which is averaged over 1000 RACs, the RACs are able to adjust quickly to the new functionality.

Secondly, we considered recovery from a fault. Initially, we programmed the RACs to implement ANDs. Then at $\tau=3000$, we randomly chose a diode and fixed its value at $d_{i}=1$ for the remainder of the simulation. This was to simulate a "stuck" fault. We see 
that initially there is a large increase in the error due to the fault, but then the adaptive algorithm was able to program around it. Note that the $\vec{c}$ that implements the AND, before and after the fault will, in general, be very different.

Finally, we considered the case of a "noisy diode". To simulate this, we again programmed a group of RACs to implement AND functions. At $\tau=3000$, we randomly chose a diode to become noisy. At each simulation time step, its value was flipped at random. This is in contrast to the stuck fault were the diode maintained a fixed value. We see that even though this diode is continually changing state, the adaptive algorithms are able to effectively neutralize it, and again implement a AND function. These results are shown in Figure 5 .

\section{SUMMARY}

In this paper, we considered programming nanocomputers with unconventional, and potentially unknown and/or random, architectures. Novel assembly processes may represents potentially inexpensive alternatives to the high costs anticipated to extend conventional design and manufacturing methods to the nanoscale. The resulting architectures may not respond to conventional computer languages. Thus, we will need new methods to program them.

To develop such methods, we introduced a simple model called a randomly assembled computer (RAC), where nano-electronic components are placed and connected at random on a chip. Input, output, and control lines connect the chip to the outside world. By manipulating the control voltages, different functions, $f$, can be implemented. Programming is achieved by minimizing an error function $E_{f}(\vec{c})$, that depended on the values of the controls $\vec{c}$. This is a potentially difficult optimization problem since in general $E_{f}$ may be a very nonlinear function in a high dimensional space. Furthermore, since the RAC was treated as a black box, the functional form for E was unknown. The only information available is the input/output pairs that resulted for a given set of controls $\vec{c}$. It should be emphasized that this model is used only for algorithm development. In this paper, we are not advocating a particular hardware or physical design. In fact, these methods are general and should be applicable to circuits with a wide range of internal structures.

We considered two methods to optimize the error function: conventional simulated an- 
nealing and a more recently developed multi-agent approach. Multi-agent optimization methods were found to be well-suited for this class of problems. These are adaptive methods based on learning algorithms. Unlike more conventional techniques, such as simulated annealing, these methods are distributed, use reinforcement learning, and have the possibility of assigning different objective functions to different optimization processes or agents. This collection of features can result in a substantial increase in convergence, especially for high dimensional problems.

One of the main question we sought to address was whether an arbitrary function can be programmed on a RAC. To help answer this question, we began by considering programming small RACs as logic gates, such as INV and XOR. Simulation results suggest that a surprisingly wide-variety of functions can be implemented on a RAC of sufficient size. For logic gates, we found that that size was on the order of $N \geq 18$ diodes to ensure a greater than $90 \%$ success rate. We found simulated annealing adequate for programming RACs of this size.

For larger RACs and more complicated functions, however, multi-agent methods were found to be essential for successful programming. We considered two-bit adders and multipliers programmed on RACs with $(\mathrm{N}=100, \mathrm{~K}=50)$. The space of functions with 4 inputs and 3 outputs is very large and picking one particular function out of that large number of possibilities is a nontrivial problem. Nonetheless, we found a large fraction of RACs could do just that using multi-agent methods. Simulated annealing, on the other hand, was shown to be not nearly as effective.

Finally, we considered issues related to fault tolerance, reprogrammability, and reliability. Electronic components at the nanoscale are expected to be very sensitive to randomness, and indeed, some degree of randomness may be unavoidable in certain types of components, such as single electron transistors. This potential intrinsic randomness could seriously impact the reliability of the components, and is thus a serious concern. RACs, on the other hand, are quite robust with respect to such randomness. The same adaptive methods that dealt with the black box nature of a RAC could adapt equally well to other potential sources of randomness. This could include intrinsic randomness of components, random assembly, as well as the unexpected appearance of faults. Furthermore, once a RAC is programmed, it 
could be as easily re-programmed, and reused potentially in a different application.

1 Tour, J.M.; Cheng, Long; Nackashi, David; Yao, Yuxing; Flat, Austen; St. Angelo, Sarah; Mallouk, Thomas; and Franzon, Paul JACS, 125, p 13279 (2003)

2 Likharev, K.K, Proc. IEEE, vol. 87, p.606, (1999)

3 Schmid, A. and Leblebici, Y., "A Modular Approach for Reliable Nanoelectronic and VeryDeep Submicron Circuit Design Based on Analog Neural Network Principles", Third IEEE Conf. Nanotechnology (2003) p.647

4 Roychowdhury, V.P.; Janes, D.B.; and Bandyopadhyay S., "Nanoelectronics Architecture for Boolean Logic", Proceedings of the IEEE, 85(4):574-588, April 1997

5 Tour J.M.; Van Zandt, W.L; Husband, C.P.; Husband, S.M.; Wilson, L.S.; Franzon, P.D. and Nackashi, D.P. IEEE Trans. Nanotechnol. 1, p 13279 (2002)

6 Wolpert, D.H.; Tumer, K.; and Bandari, E. Phys. Rev. E, Vol. 69, pg. 017701, (2004)

7 Wolpert, D.H. and Millonas, M., NASA Technical Report

8 Wolpert, D.H.; Wheeler, K.; and Tumer, K. Europhysics Letters, Vol. 49, No. 6, (2000)

9 Wolpert, D.H. and Tumer, K., "An Introduction to Collective Intelligence" in Handbook of Agent technology, J.M. Bradshow, ed., AAAI Press/MIT Press, (1999)

10 Wolpert, D.H. and Tumer, K., Advances in Complex Systems, 4, p 265, (2001)

11 Sutton, R. S. and Barto, A. G., Reinforcement Learning: An Introduction, MIT Press, Cambridge, MA, (1998)

12 Wolpert, D.H., "Information theory - the bridge connecting bounded rational game theory and statistical physics", in Complex Engineering ystems, D. Braha and Y. Bar-Yam (Ed.'s), Perseus books, in press

13 Wolpert, D.H., and Bieniawski, S., "Distributed Control by Lagrangian Steepest Descent", Proceedings of IEEE 2004 Conference on Decision and Control, in press 


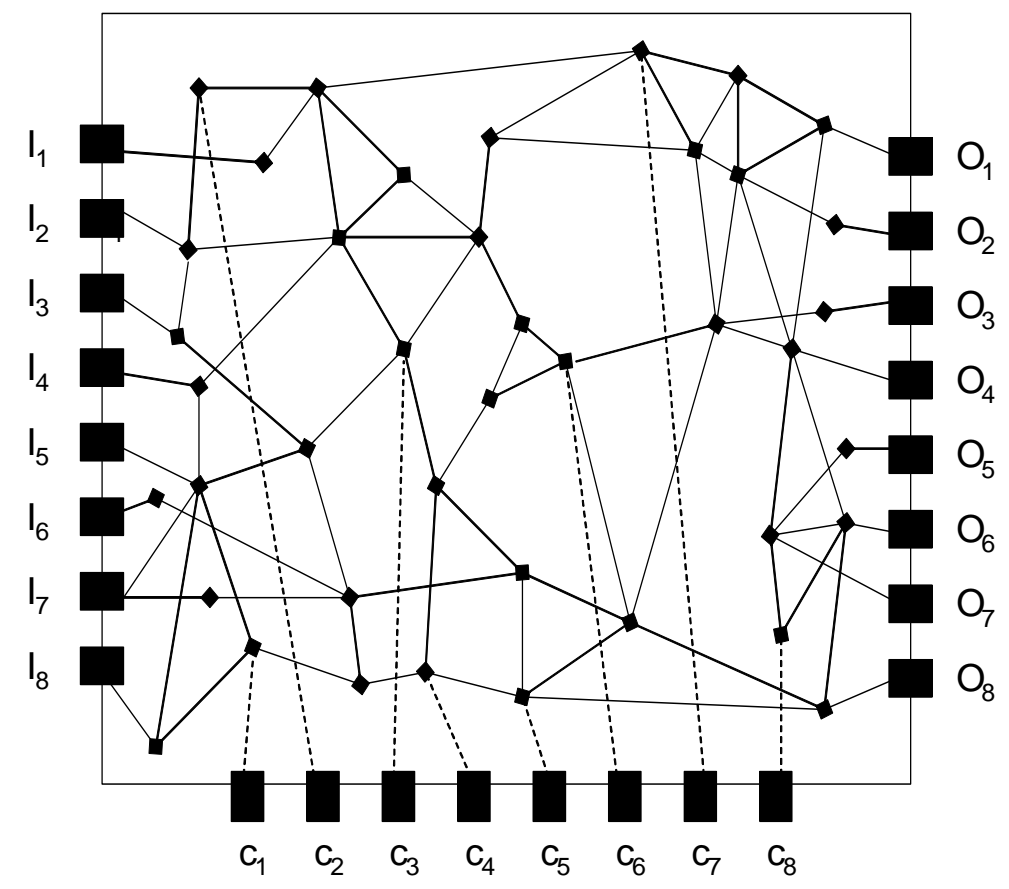

FIG. 1: Schematic of a Randomly Assembled Computer (RAC). Diodes are placed at random on a chip and connected with random strengths $\omega_{i j}$. Connections to the outside world is made via pads representing inputs, outputs, and controls. By manipulating control voltages, different functions can be implemented. 

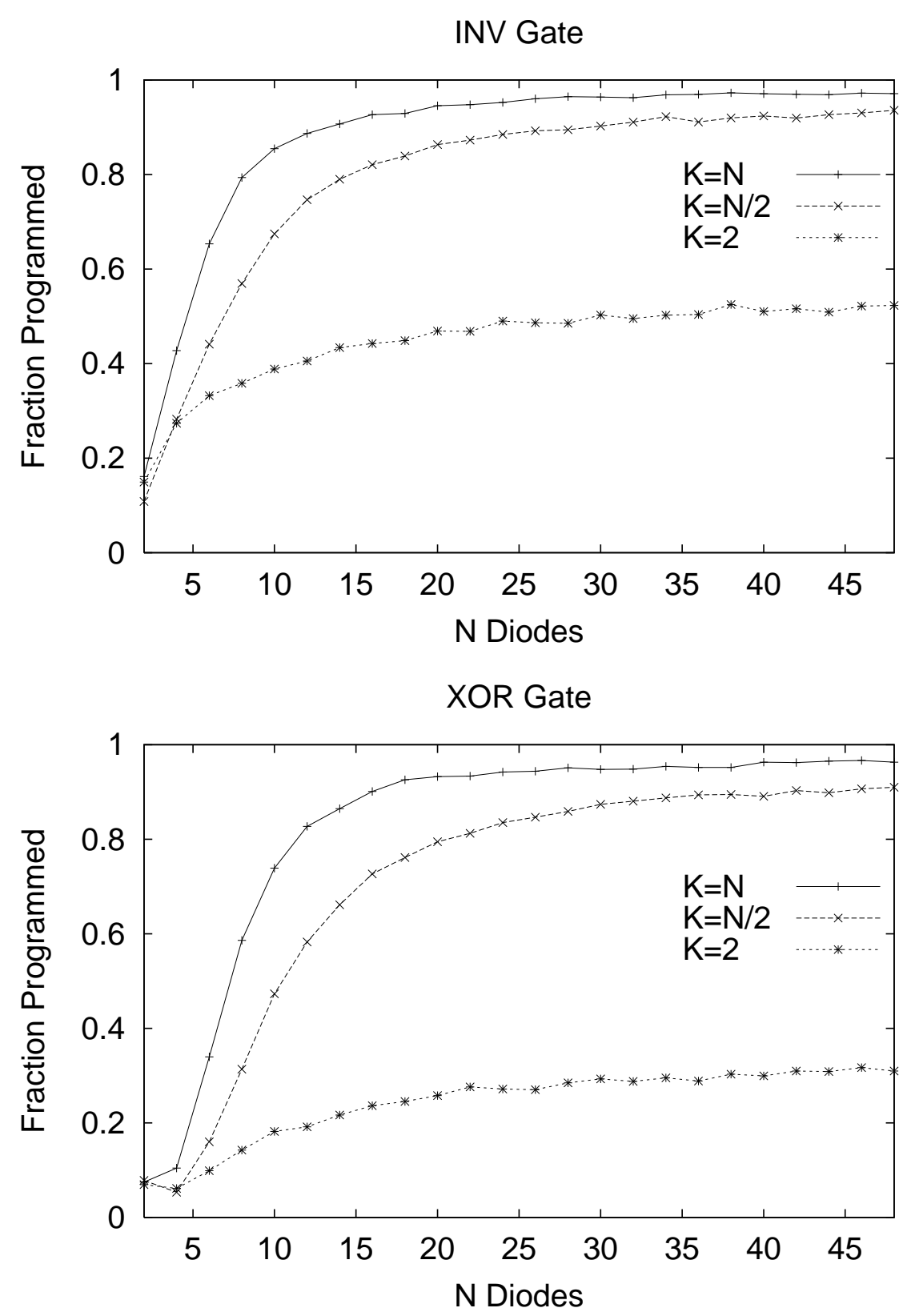

FIG. 2: Fraction of RACs with $N$ diodes and $K$ controls successfully programmed to implement INV and XOR function. The fraction is out of an ensemble of 10,000 RACs. Simulated annealing was used for the programming. 


\begin{tabular}{|ll|lllll|lll|}
\hline$I_{1}$ & $I_{2}$ & $I_{1}$ & + & $I_{2}$ & $I_{1}$ & $*$ & $I_{2}$ \\
\hline 0 & 0 & 0 & 0 & 0 & 0 & 0 & 0 & 0 & 0 \\
0 & 0 & 0 & 1 & 0 & 0 & 1 & 0 & 0 & 0 \\
0 & 0 & 1 & 0 & 0 & 1 & 0 & 0 & 0 & 0 \\
0 & 0 & 1 & 1 & 0 & 1 & 1 & 0 & 0 & 0 \\
0 & 1 & 0 & 0 & 0 & 0 & 1 & 0 & 0 & 0 \\
0 & 1 & 0 & 1 & 0 & 1 & 0 & 0 & 0 & 1 \\
0 & 1 & 1 & 0 & 0 & 1 & 1 & 0 & 1 & 0 \\
0 & 1 & 1 & 1 & 1 & 0 & 0 & 0 & 1 & 1 \\
1 & 0 & 0 & 0 & 0 & 1 & 0 & 0 & 0 & 0 \\
1 & 0 & 0 & 1 & 0 & 1 & 1 & 0 & 1 & 0 \\
1 & 0 & 1 & 0 & 1 & 0 & 0 & 1 & 0 & 0 \\
1 & 0 & 1 & 1 & 1 & 0 & 1 & 1 & 1 & 0 \\
1 & 1 & 0 & 0 & 0 & 1 & 1 & 0 & 0 & 0 \\
1 & 1 & 0 & 1 & 1 & 0 & 0 & 0 & 1 & 1 \\
1 & 1 & 1 & 0 & 1 & 0 & 1 & 1 & 1 & 0 \\
1 & 1 & 1 & 1 & 1 & 1 & 0 & 0 & 0 & 1 \\
\hline
\end{tabular}

TABLE I: Truth table for a 2-Bit Adder $\left(O=I_{1}+I_{2}\right)$ and a 2-Bit Multiplier $\left(O=I_{1} * I_{2}\right)$ 

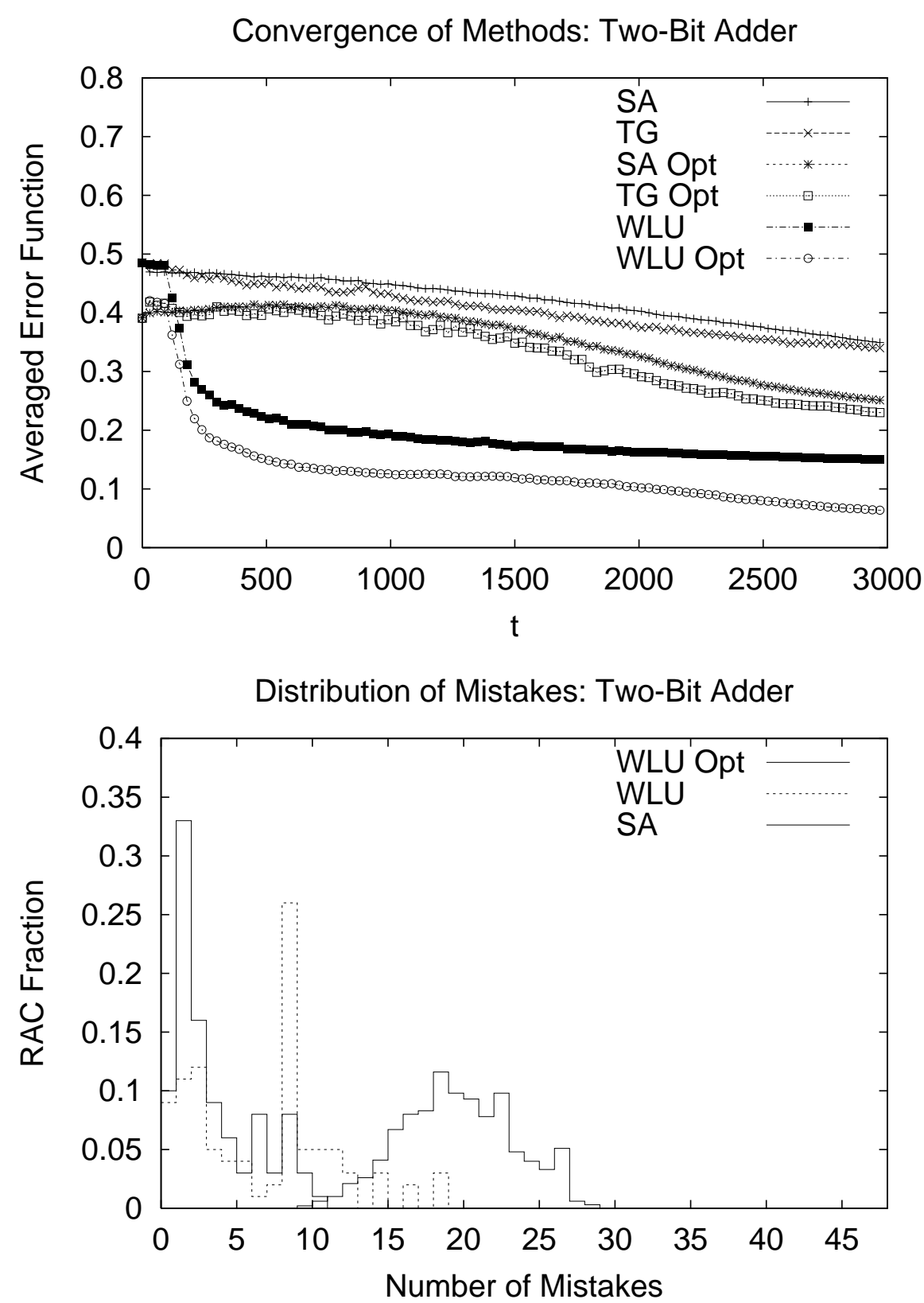

FIG. 3: Two-Bit Adders with carry with $\mathrm{N}=100$ diodes, $\mathrm{K}=40$ controls, and $\mathrm{T}=5$ iterations. Top plot compares the convergence of the error function averaged over 1000 RACs using different programming methods. Bottom plot gives the fraction of RACs making a given number of mistakes (out of 48 possible) after programming. WLU Opt performs the best with $10 \%$ making no mistakes and $33 \%$ making one mistake. WLU is next with $9 \%, 11 \%$, and $12 \%$ making 0,1 , and 3 mistakes. The best result for $\mathrm{SA}$ is $0.2 \%$ making 9 mistakes. 

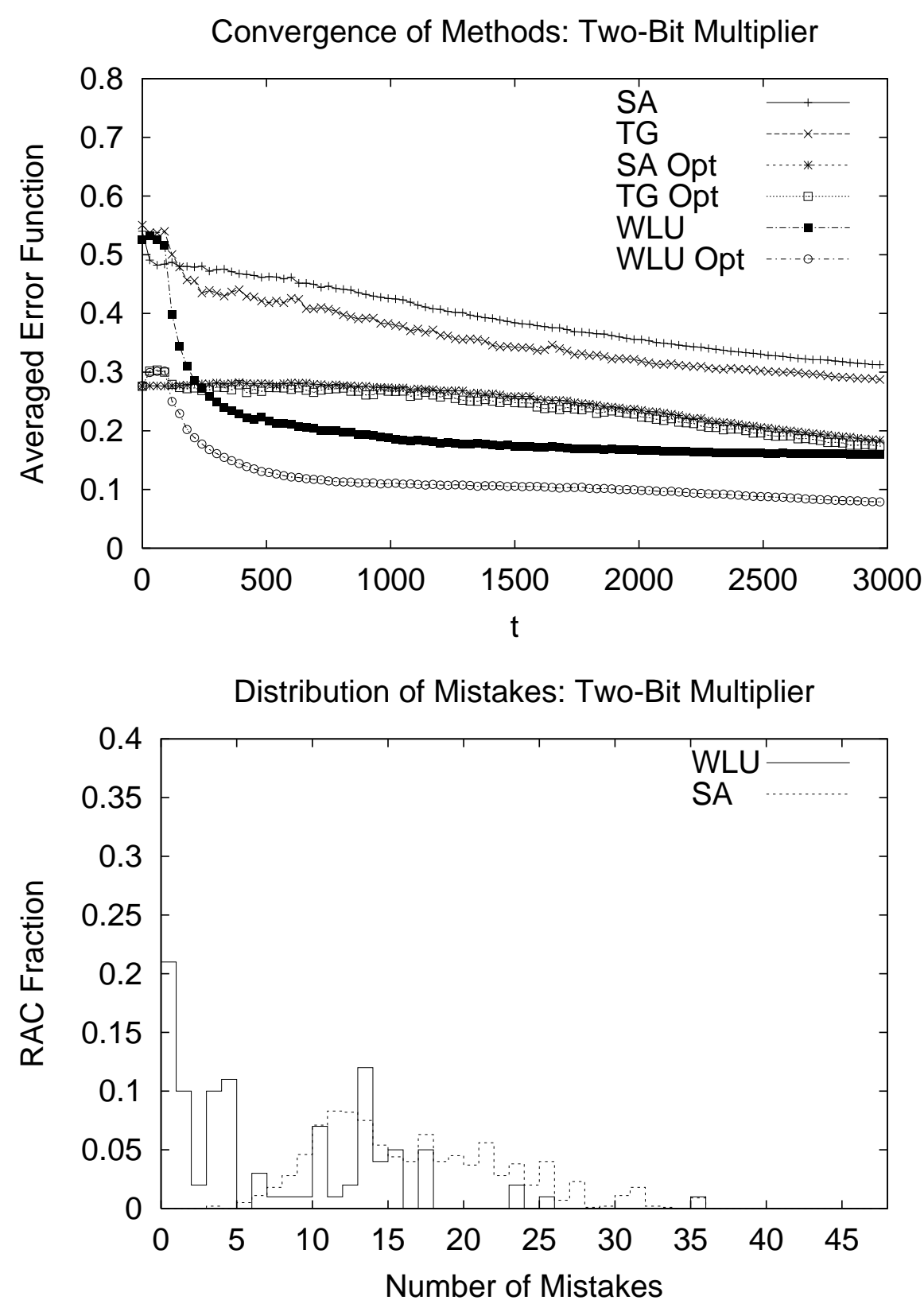

FIG. 4: Two-Bit Multiplier with $\mathrm{N}=100$ diodes, $\mathrm{K}=40$ controls, and $\mathrm{T}=5$ iterations. WLU programs $21 \%$ with no mistakes and SA's best effort is 3 mistakes. In this case, WLU Opt has a smaller fraction than WLU making zero mistakes at $9 \%$ even though its ensemble averaged error function is lower. For clarity, only WLU and SA are shown. 

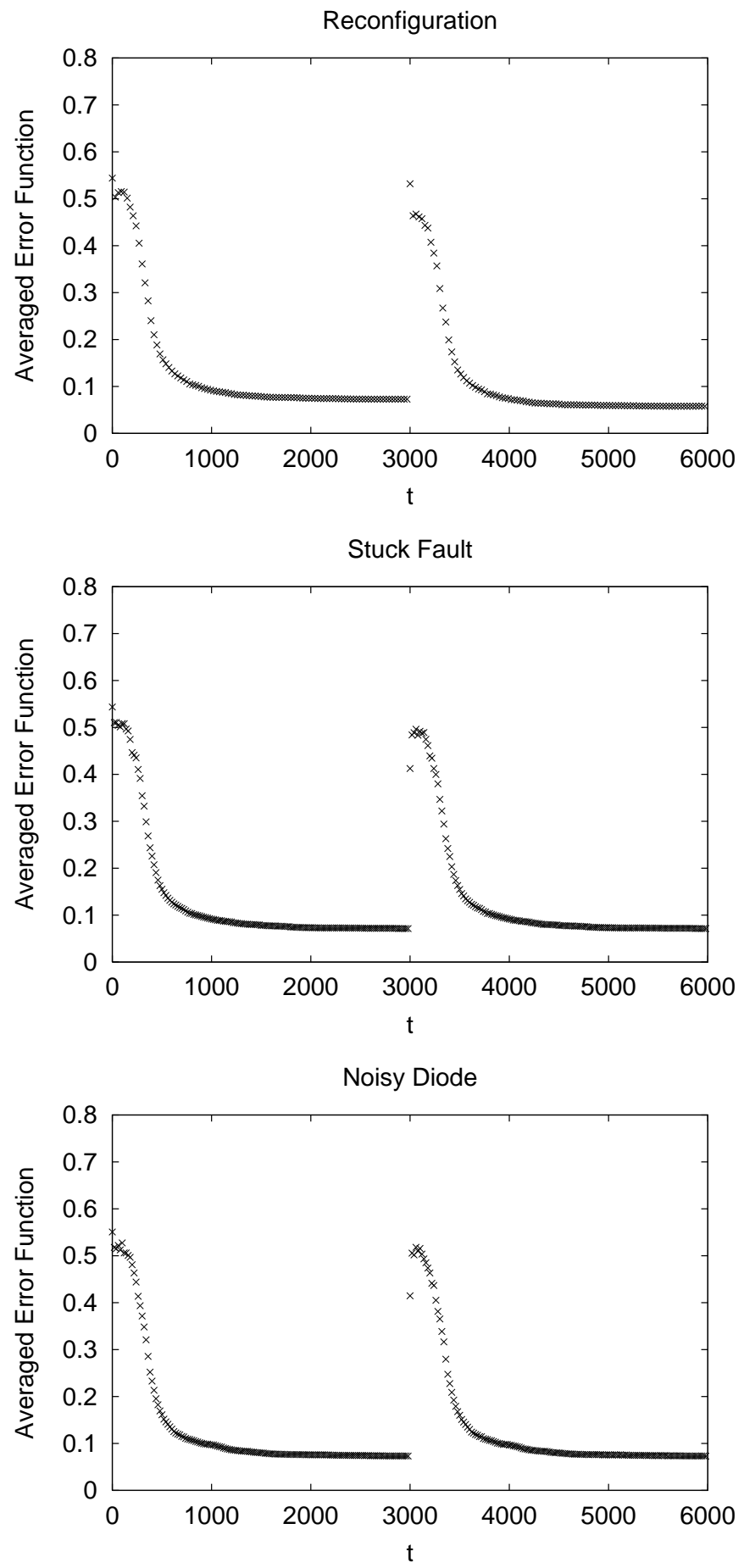

FIG. 5: Adaptive approach to fault tolerance and reconfiguration. Error functions is averaged over 1000 RACs with $(\mathrm{N}=20, \mathrm{~K}=10)$. An AND function is initially implemented. At $\mathrm{t}=3000$, the system is perturbed. In the top plot, the RACs are reconfigured to implement an XOR function. In the middle plot, a "stuck" fault is introduced, and in the bottom plot, the RACs develop a "noisy" component. In each case, the RACs adjusts to the new situation. 